\title{
Noncommutative irreducible characters of the symmetric group and noncommutative Schur functions
}

\author{
Stephanie VAn WilligenburG*
}

\begin{abstract}
In the Hopf algebra of symmetric functions, Sym, the basis of Schur functions is distinguished since every Schur function is isomorphic to an irreducible character of a symmetric group under the Frobenius characteristic map. In this note we show that in the Hopf algebra of noncommutative symmetric functions, NSym, of which Sym is a quotient, the recently discovered basis of noncommutative Schur functions exhibits that every noncommutative Schur function is isomorphic to a noncommutative irreducible character of a symmetric group when working in noncommutative character theory. We simultaneously show that a second basis of NSym consisting of Young noncommutative Schur functions also satisfies that every element is isomorphic to a noncommutative irreducible character of a symmetric group.
\end{abstract}

AMS 2010 SUBJECT CLASSIFICATIONS: Primary 05E05, 16T30; secondary 05E10, 16T05, 20B30, 20C30, 33D52.

KEYWORDS AND PHRASES: Descent algebra, irreducible character, noncommutative character theory, noncommutative symmetric function, Schur function, symmetric group.

\section{Introduction}

In the representation theory of the symmetric group it is long-established [24] that every Schur function is isomorphic to an irreducible character of a symmetric group under the Frobenius characteristic map. Since this was established, Schur functions have continued to be a vibrant area of research due to their appearance in areas such as enumerative combinatorics through Young tableaux, plus areas such as algebraic geometry and diagonal harmonics. Throughout, Schur functions have exhibited beautiful combinatorial

arXiv: 1210.6013

*The author was supported in part by the National Sciences and Engineering Research Council of Canada. 
properties such as Pieri rules, the Littlewood-Richardson rule, and Kostka numbers.

Schur functions also form a basis for the Hopf algebra of symmetric functions, Sym, which is contained in the Hopf algebra of quasisymmetric functions, QSym. Recently a new basis for QSym has been discovered [13], using the combinatorics of Macdonald polynomials [12]. This new basis was termed the basis of quasisymmetric Schur functions as quasisymmetric Schur functions refine Schur functions in a natural way. Furthermore, both the basis of quasisymmetric Schur functions and their dual basis, the basis of noncommutative Schur functions in the Hopf algebra of noncommutative symmetric functions NSym, exhibit many natural generalisations of combinatorial properties of Schur functions such as Pieri rules [13, Theorem 6.3], the Littlewood-Richardson rule [3, 14] and Kostka numbers [13, Theorem 6.1].

Therefore a pertinent question to ask is can every noncommutative Schur function be realized as being isomorphic to an irreducible character of a symmetric group? In this note we answer the question in the affirmative in Theorem 6.2 using noncommutative character theory.

The theory of noncommutative character theory for the symmetric groups was initiated in [25]. It was then developed independently by Malvenuto and Reutenauer [19] and Gelfand et al. [11], and brought together by Jöllenbeck [15]. Other papers on the subject have also been written including [1, 4, $6,8,10,16,20,22]$. This theory is more amenable to combinatorial manipulation involving permutations, and many classical results such as the Murnaghan-Nakayama rule, the Littlewood-Richardson rule and results on Foulkes characters are more straightforward to obtain using this theory. An exposition can be found in the monograph [5] by Blessenohl and Schocker, which will serve as our primary reference for this theory later.

With this in mind, we will spend the remainder of this note working towards establishing Theorem 6.2, which in essence states the following.

Theorem. Every noncommutative Schur function is isomorphic to a noncommutative irreducible character of a symmetric group.

At the same time, we will establish the following for another basis of NSym, called the basis of Young noncommutative Schur functions, whose combinatorics is related to that of Young tableaux.

Theorem. Every Young noncommutative Schur function is isomorphic to a noncommutative irreducible character of a symmetric group. 


\section{Combinatorial tools}

We begin by recalling the combinatorial concepts of compositions and partitions, which will be used to index the variety of functions we will subsequently encounter. A composition is a finite list of positive integers, while a partition is a finite unordered list of positive integers, which we list in weakly decreasing order for convenience. In both cases the integers are called parts. We say a composition (respectively, partition) $\alpha$ is a composition (respectively, partition) of $n$, denoted by $\alpha \vDash n$ (respectively, $\alpha \vdash n$ ), if the parts of $\alpha$ sum to $n$. We denote by 0 both the empty composition and empty partition of 0 . The underlying partition of a composition $\alpha$, denoted by $\widetilde{\alpha}$, is the partition obtained from $\alpha$ by reordering the parts of $\alpha$ into weakly decreasing order.

One composition closely related to a composition $\alpha=\left(\alpha_{1}, \ldots, \alpha_{\ell}\right)$ is the reversal of $\alpha$, denoted by $\alpha^{*}$, given by $\alpha^{*}=\left(\alpha_{\ell}, \ldots, \alpha_{1}\right)$. We say a composition $\beta$ refines a composition $\alpha$ if we can obtain the parts of $\alpha$ in order by adding together adjacent parts of $\beta$ in order, denoted by $\beta \preccurlyeq \alpha$. For any composition $\alpha \vDash n$, say $\alpha=\left(\alpha_{1}, \ldots, \alpha_{\ell}\right)$, we associate the unique subset $\operatorname{Set}(\alpha) \subseteq[n-1]=\{1,2, \ldots, n-1\}$ given by the partial sums of $\alpha$, that is,

$$
\operatorname{Set}(\alpha)=\left\{\sum_{i=1}^{k} \alpha_{i} \mid 1 \leqslant k<\ell\right\} .
$$

Conversely, for $J \subseteq[n-1]$ we write $\operatorname{comp}(J)=\alpha$ if $\operatorname{Set}(\alpha)=J$.

Example 2.1. If $\alpha=(2,4,1,2)$, then $\widetilde{\alpha}=(4,2,2,1), \alpha^{*}=(2,1,4,2)$, $(2,1,1,3,1,1) \preccurlyeq(2,1,4,2)$ and $\operatorname{Set}(\alpha)=\{2,6,7\} \subset[8]$.

Now we use compositions to create diagrams.

Definition 2.2. Given a composition $\alpha=\left(\alpha_{1}, \ldots, \alpha_{\ell}\right) \vDash n$, we say the composition diagram of $\alpha$, also denoted by $\alpha$, is the left-justified array of $n$ cells with $\alpha_{i}$ cells in the $i$-th row from the bottom. The cell in the $i$-th row from the bottom and $j$-th cell from the left is denoted by the pair $(i, j)$.

Our next task is to fill our composition diagrams with integers.

Definition 2.3. Given a composition $\alpha$, a standard Young composition tableau (abbreviated to SYCT) $\tau$ of shape $\alpha$, denoted by $\operatorname{sh}(\tau)=\alpha$, is a bijective filling of the cells of $\alpha$

$$
\tau: \alpha \rightarrow[n]
$$

such that 
1. the entries in each row are increasing when read from left to right,

2. the entries in the first column are increasing when read from the row with the smallest index to the row with the largest index,

3. if $i<j$ and $\tau(j, k)<\tau(i, k+1)$ then $\tau(j, k+1)$ exists and $\tau(j, k+1)<$ $\tau(i, k+1)$.

We use SYCTs to denote the set of all SYCTs where the context is clear. The descent set of an SYCT $\tau$ is defined to be

$\operatorname{Des}(\tau)=\{i \mid i+1$ appears in the same column or a column to the left of $i\}$.

Given an $\operatorname{SYCT} \tau$ with $n$ cells, we let $\operatorname{comp}(\tau)=\operatorname{comp}(\operatorname{Des}(\tau)) \vDash n$ and call it the descent composition of $\tau$.

Example 2.4. An SYCT $\tau$ with $\operatorname{sh}(\tau)=(2,4,1,2), \operatorname{Des}(\tau)=\{1,3,4,6\}$ and $\operatorname{comp}(\tau)=(1,2,1,2,3)$.

\begin{tabular}{|l|l|l|l|}
\hline 7 & 8 & \multicolumn{2}{|c}{} \\
\hline 5 & \multicolumn{3}{|c}{} \\
\hline 2 & 3 & 6 & 9 \\
\hline 1 & 4 & \multicolumn{2}{|c}{} \\
\cline { 1 - 2 } &
\end{tabular}

Remark 2.5. There is a natural bijection $\hat{\rho}$ from the set of all SYCTs to standard Young tableaux that arise in the classical representation theory of the symmetric group: Given an SYCT $\tau$ we obtain $\hat{\rho}(\tau)$ by sorting the entries in each column from bottom to top in increasing order and then bottom justifying the columns. For further details, see [17]. This bijection is analogous to the bijection due to Mason [21] for standard reverse tableaux.

It is well-known that the symmetric group $\mathfrak{S}_{n}$ is the group of bijections $\sigma:[n] \rightarrow[n]$, called permutations, which we will denote in one-line notation $\sigma(1) \cdots \sigma(n)$. The symmetric group $\mathfrak{S}_{n}$ is also known as the Coxeter group of type $A, A_{n-1}$, whose standard generators are $s_{i}=1 \cdots(i-1)(i+1) i(i+$ $2) \cdots n$ for $1 \leqslant i \leqslant n-1$.

The descent set of a permutation $\sigma \in \mathfrak{S}_{n}$ is defined to be

$$
\operatorname{Des}(\sigma)=\{i \in[n-1] \mid \sigma(i)>\sigma(i+1)\}
$$

In terms of Coxeter groups $i \in \operatorname{Des}(\sigma)$ if and only if there is some minimal length word in terms of standard generators for $\sigma$ that ends in $s_{i}$. Given a permutation $\sigma \in \mathfrak{S}_{n}$ we let $\operatorname{comp}(\sigma)=\operatorname{comp}(\operatorname{Des}(\sigma)) \vDash n$ and call it the descent composition of $\sigma$. 
We now review a number of algebraic concepts. Throughout we let $\mathbb{K}$ denote our coefficient ring, which under minimal conditions we can take to be $\mathbb{Z}$ but which we ordinarily take to be $\mathbb{C}$.

\section{The Hopf algebras of symmetric and quasisymmetric functions}

We now recap some pertinent facts about the Hopf algebra of symmetric functions. Further details can be found in excellent texts such as [9, 18, 23, 26]. The Hopf algebra of symmetric functions is a graded Hopf algbera

$$
\operatorname{Sym}=\bigoplus_{n \geqslant 0} \operatorname{Sym}^{n} \subseteq \mathbb{K}\left[\left[x_{1}, x_{2}, \ldots\right]\right]
$$

and the fundamental theorem of symmetric functions states that $S y m$ is a polynomial algebra in the elementary symmetric functions:

$$
S y m \cong \mathbb{K}\left[e_{1}, e_{2}, \ldots\right]
$$

where the $r$-th elementary symmetric function for $r>0$ is

$$
e_{r}=\sum_{1 \leqslant i_{1}<i_{2}<\cdots<i_{r}} x_{i_{1}} x_{i_{2}} \cdots x_{i_{r}} .
$$

It is also a polynomial algebra in the complete homogeneous symmetric functions:

$$
\operatorname{Sym} \cong \mathbb{K}\left[h_{1}, h_{2}, \ldots\right]
$$

where the $r$-th complete homogeneous symmetric function for $r>0$ is

$$
h_{r}=\sum_{1 \leqslant i_{1} \leqslant i_{2} \leqslant \cdots \leqslant i_{r}} x_{i_{1}} x_{i_{2}} \cdots x_{i_{r}}=\sum_{\left(\alpha_{1}, \ldots, \alpha_{\ell}\right) \models r}(-1)^{\ell-r} e_{\alpha_{1}} \cdots e_{\alpha_{\ell}} .
$$

To obtain $\mathbb{K}$-bases for Sym define for a partition $\lambda=\left(\lambda_{1}, \ldots, \lambda_{\ell}\right)$ the elementary and complete homogeneous symmetric functions to be, respectively,

$$
\begin{aligned}
e_{\lambda} & =e_{\lambda_{1}} \cdots e_{\lambda_{\ell}} \\
h_{\lambda} & =h_{\lambda_{1}} \cdots h_{\lambda_{\ell}} .
\end{aligned}
$$

From here the fundamental theorem of symmetric functions implies that $\operatorname{Sym}^{n}$ for $n>0$ has either $\left\{e_{\lambda}\right\}_{\lambda \vdash n}$ or $\left\{h_{\lambda}\right\}_{\lambda \vdash n}$ as a $\mathbb{K}$-basis. We define $e_{0}=h_{0}=1$ and $e_{r}=h_{r}=0$ for $r<0$. 
However, the most renowned $\mathbb{K}$-basis for Sym is that consisting of Schur functions, which can be defined in a myriad of ways including as quotients, as irreducible representations of a symmetric group, or as generating functions for tableaux, but for our purposes we will define them using the following Jacobi-Trudi determinant.

Definition 3.1. Given a partition $\lambda=\left(\lambda_{1}, \ldots, \lambda_{\ell}\right)$ the Schur function $s_{\lambda}$ is defined to be $s_{0}=1$ and otherwise

$$
s_{\lambda}=\operatorname{det}\left(h_{\lambda_{i}-i+j}\right)_{i, j=1}^{\ell}
$$

Again we have $\left\{s_{\lambda}\right\}_{\lambda \vdash n}$ is a $\mathbb{K}$-basis for $S y m^{n}$.

The Hopf algebra of symmetric functions $S y m$ is contained in the graded Hopf algebra of quasisymmetric functions QSym

$$
Q \operatorname{Sym}=\bigoplus_{n \geqslant 0} Q \operatorname{Sym}^{n} \subseteq \mathbb{K}\left[\left[x_{1}, x_{2}, \ldots\right]\right]
$$

where $Q \operatorname{Sym}^{n}$ for $n>0$ is spanned by the $\mathbb{K}$-basis of monomial quasisymmetric functions $\left\{M_{\alpha}\right\}_{\alpha \vDash n}$ given by

$$
M_{\alpha}=\sum_{i_{1}<\cdots<i_{\ell}} x_{i_{1}}^{\alpha_{1}} \cdots x_{i_{\ell}}^{\alpha_{\ell}}
$$

and is also spanned by the $\mathbb{K}$-basis of fundamental quasisymmetric functions $\left\{F_{\alpha}\right\}_{\alpha \models n}$ given by

$$
F_{\alpha}=\sum_{\beta \preccurlyeq \alpha} M_{\beta}
$$

and $M_{0}=F_{0}=1$.

In [17] another $\mathbb{K}$-basis for $Q S y m^{n}$ was introduced, that of Young quasisymmetric Schur functions $\left\{\hat{\mathcal{S}}_{\alpha}\right\}_{\alpha \vDash n}$ defined combinatorially as follows.

Definition 3.2. [17, Proposition 5.2.2] Let $\alpha \vDash n$. Then the Young quasisymmetric Schur function $\hat{\mathcal{S}}_{\alpha}$ is given by $\hat{\mathcal{S}}_{0}=1$ and otherwise

$$
\hat{\mathcal{S}}_{\alpha}=\sum_{\beta} \hat{d}_{\alpha \beta} F_{\beta}
$$

where the sum is over all compositions $\beta \vDash n$ and $\hat{d}_{\alpha \beta}=$ the number of $S Y C T s \tau$ of shape $\alpha$ such that $\operatorname{Des}(\tau)=\operatorname{Set}(\beta)$. 
This basis is related to the $\mathbb{K}$-basis for $Q S y m^{n}$ known as the basis of quasisymmetric Schur functions $\left\{\check{\mathcal{S}}_{\alpha}\right\}_{\alpha \models n}[3,13,14]$ by the involutive automorphism of QSym that maps $F_{\alpha} \mapsto F_{\alpha^{*}}$, which maps $\hat{\mathcal{S}}_{\alpha} \mapsto \check{\mathcal{S}}_{\alpha^{*}}$. Thus, quasisymmetric Schur functions can be defined combinatorially as follows.

Definition 3.3. Let $\alpha \vDash n$. Then the quasisymmetric Schur function $\check{\mathcal{S}}_{\alpha}$ is given by $\check{\mathcal{S}}_{0}=1$ and otherwise

$$
\check{\mathcal{S}}_{\alpha}=\sum_{\beta} \hat{d}_{\alpha^{*} \beta^{*}} F_{\beta}
$$

where the sum is over all compositions $\beta \vDash n$ and $\hat{d}_{\alpha \beta}=$ the number of SYCTs $\tau$ of shape $\alpha$ such that $\operatorname{Des}(\tau)=\operatorname{Set}(\beta)$.

Example 3.4. We have $\hat{\mathcal{S}}_{(1,3)}=F_{(1,3)}$ from the SYCT

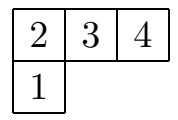

while $\check{\mathcal{S}}_{(1,3)}=F_{(1,3)}+F_{(2,2)}$ from the following SYCTs.

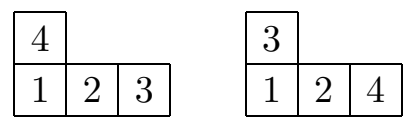

Remark 3.5. These functions are named (Young) quasisymmetric Schur functions because they refine Schur functions in a natural way, that is,

$$
s_{\lambda}=\sum_{\widetilde{\alpha}=\lambda} \hat{\mathcal{S}}_{\alpha}=\sum_{\widetilde{\alpha}=\lambda} \check{\mathcal{S}}_{\alpha}
$$

and additionally refine many properties of Schur functions $[3,13,14,17]$.

\section{Classical character theory}

The basics of representation theory for finite groups can be found in texts such as [7], and for the symmetric groups in particular in texts such as [23]. For a finite group $G$, a finite dimensional representation of $G$ is a group homomorphism $\rho: G \rightarrow G l(V)$ for some finite dimensional $\mathbb{K}$-vector space $V$. We can identify $\rho$ with $V$ viewed as a $G$-module under the group action

$$
g \cdot v=\rho(g)(v) \quad \forall g \in G, v \in V .
$$


The character of $\rho$ is the map $G \rightarrow \mathbb{K}$ given by the trace function, $\operatorname{tr}(\rho(g))$. The set of irreducible characters of $G$ (that is, the characters of the irreducible modules) is denoted $\operatorname{Irr}(G)$, and every character of $G$ is a positive $\mathbb{Z}$-linear combination of irreducible characters. Since non-isomorphic irreducible $G$-modules have distinct characters, $\operatorname{Irr}(G)$ is in bijection with the set of isomorphism classes of irreducible $G$-modules.

The Grothendieck ring $\mathcal{R}(G)$ of finite dimensional representations of $G$ has as a basis the set of isomorphism classes of irreducible $G$-modules, that is $\mathcal{R}(G) \cong \mathbb{K} \operatorname{Irr}(G)$ as a vector space, with operations

$$
[M]+[N]=[M \oplus N], \quad \text { and } \quad[M] *[N]=[M \otimes N] .
$$

The algebra of class functions of $G$ is

$$
\mathcal{C} \ell(G)=\{\phi: G \rightarrow \mathbb{K} \mid \phi \text { constant on conjugacy classes of } G\},
$$

under pointwise addition and multiplication. The set $\operatorname{Irr}(G)$ forms a $\mathbb{Z}$-basis of $\mathcal{C} \ell(G)$. In fact $\mathcal{R}(G) \cong \mathcal{C} \ell(G)$, the isomorphism being given by the trace function, $\operatorname{tr}: \mathcal{R}(G) \rightarrow \mathcal{C} \ell(G)$.

In the case of the symmetric groups, the conjugacy classes of $\mathfrak{S}_{n}$ are indexed by partitions $\lambda \vdash n$ corresponding to the cycle types of permutations. Hence the irreducible modules, called Specht modules, $S^{\lambda}$ of $\mathfrak{S}_{n}$ are also indexed by partitions $\lambda \vdash n$. We denote the irreducible characters by $\chi^{\lambda}=\operatorname{tr}\left(S^{\lambda}\right)$. There is a natural embedding of $\mathfrak{S}_{m} \times \mathfrak{S}_{n}$ into $\mathfrak{S}_{m+n}$, and so considering the direct sum of their representation rings,

$$
\mathcal{R}=\bigoplus_{n \geqslant 0} \mathcal{R}\left(\mathfrak{S}_{n}\right),
$$

one can define a graded outer product on $\mathcal{R}$ using induction, that is,

$$
[M] \cdot[N]=\left[\operatorname{Ind} \uparrow \mathfrak{S}_{\mathfrak{S}_{m} \times \mathfrak{S}_{n}}^{\mathfrak{I}_{m}} M \otimes N\right] \quad \text { for }[M] \in \mathcal{R}\left(\mathfrak{S}_{m}\right),[N] \in \mathcal{R}\left(\mathfrak{S}_{n}\right) .
$$

By identification via the trace function, this induces an isomorphic algebraic structure on the direct sum of class algebras,

$$
\mathcal{C} \ell=\bigoplus_{n \geqslant 0} \mathcal{C} \ell\left(\mathfrak{S}_{n}\right)
$$

It is well-known [23, Section 4.7] that $\mathcal{C} \ell$ is isomorphic to Sym, the algebra of symmetric functions, the isomorphism being given by the Frobenius 
characteristic map ch $: \mathcal{C} \ell \rightarrow S y m$ determined by

$$
\operatorname{ch}\left(\chi^{\lambda}\right)=s_{\lambda}
$$

that is, the irreducible character indexed by $\lambda$ is mapped to the Schur function indexed by $\lambda$.

The characteristic map can also be described in terms of Young characters [5, p. 6]. Let $\alpha=\left(\alpha_{1}, \ldots, \alpha_{\ell}\right) \vDash n$ and $\alpha=\operatorname{comp}(J)$, where $J \subseteq[n-1]$. Letting $S=\left\{s_{1}, \ldots, s_{n-1}\right\}$ be the set of standard generators for $W=\mathfrak{S}_{n}$ as a Coxeter group, we let $J^{c}$ be the complement of $J$ in $[n-1]$, and let $W_{\alpha}=W_{J^{c}}$ be the Young subgroup of $\mathfrak{S}_{n}$ generated by $\left\{s_{j} \mid j \in J^{c}\right\}$, which we identify with $\mathfrak{S}_{\alpha_{1}} \times \cdots \times \mathfrak{S}_{\alpha_{\ell}}$. The Young character $\xi^{\alpha}$ is defined to be the character of $\mathfrak{S}_{n}$ induced by the trivial character of $W_{\alpha}$, that is,

$$
\xi^{\alpha}=\operatorname{Ind} \uparrow_{W_{\alpha}}^{\mathfrak{S}_{n}} 1 .
$$

Note that $\xi^{\alpha}=\xi^{\beta}$ if $\widetilde{\alpha}=\widetilde{\beta}$, that is, if $\alpha$ and $\beta$ have the same underlying partition. Furthermore, $\operatorname{ch}\left(\xi^{\alpha}\right)=h_{\lambda}$, where $\lambda=\widetilde{\alpha}$ and $h_{\lambda}$ is the complete homogeneous symmetric function indexed by $\lambda[18$, p. 114] .

\section{The permutation and descent algebras, and the Hopf algebra of noncommutative symmetric functions}

For any Coxeter group $W$, Solomon [25] defined a subalgebra of the group algebra called the descent algebra of $W$. For the symmetric group $\mathfrak{S}_{n}$, we denote its group algebra by $\mathcal{P}_{n}=\mathbb{K} \widetilde{S}_{n}$, and its descent algebra $\mathcal{D}_{n} \subset \mathcal{P}_{n}$. To any $I \subseteq[n-1]$ we associate the element $B_{I} \in \mathcal{P}_{n}$ (respectively, $D_{I} \in \mathcal{P}_{n}$ ) that is the formal sum of all permutations in $\mathfrak{S}_{n}$ whose descent set is contained in (respectively, is equal to) $I$, that is,

$$
B_{I}=\sum_{\operatorname{Des}(\sigma) \subseteq I} \sigma, \quad D_{I}=\sum_{\operatorname{Des}(\sigma)=I} \sigma .
$$

Then $\left\{B_{I}\right\}_{I \subseteq[n-1]}$ and $\left\{D_{I}\right\}_{I \subseteq[n-1]}$ respectively are $\mathbb{Z}$-bases of $\mathcal{D}_{n}$. For ease of computation we write $B_{\alpha}=B_{I}$ and $D_{\alpha}=D_{I}$ when $n$ is understood from context and $\operatorname{comp}(I)=\alpha$. If we let $W^{\alpha}$ denote the set of minimal length representatives of the left cosets of $W_{\alpha}$ in $\mathfrak{S}_{n}$, then note that $W^{\alpha}=\{\pi \in$ $\left.\mathfrak{S}_{n} \mid \operatorname{Des}(\pi) \subseteq \operatorname{Set}(\alpha)\right\}$. Thus $B_{\alpha}=\sum_{\sigma \in W^{\alpha}} \sigma$.

Using the convention that $\mathcal{D}_{0}=\mathcal{P}_{0}=\mathbb{K}$, we write

$$
\mathcal{P}=\bigoplus_{n \geqslant 0} \mathcal{P}_{n}, \quad \text { and } \quad \mathcal{D}=\bigoplus_{n \geqslant 0} \mathcal{D}_{n} .
$$


In relation to the representation theory of the 0-Hecke algebra we have the Hopf algebra of noncommutative symmetric functions, and a summary of connections can be found in [27]. We describe it here in order to both algebraically and combinatorially define two key objects of study, noncommutative Schur functions and Young noncommutative Schur functions.

The Hopf algebra of noncommutative symmetric functions [11] is a graded Hopf algebra

$$
\text { NSym }=\bigoplus_{n \geqslant 0} N S y m^{n} \cong \mathbb{K}\left\langle\mathbf{e}_{1}, \mathbf{e}_{2}, \ldots\right\rangle
$$

where we set $\mathbf{e}_{0}=1$ and the $\mathbf{e}_{r}$ for $r>0$ are noncommuting indeterminates of degree $r$. We call $\mathbf{e}_{r}$ the $r$-th noncommutative elementary symmetric function. Defining the $r$-th noncommutative complete homogeneous symmetric function to be

$$
\mathbf{h}_{r}=\sum_{\left(\beta_{1}, \beta_{2}, \ldots, \beta_{m}\right) \models r}(-1)^{m-n} \mathbf{e}_{\beta_{1}} \mathbf{e}_{\beta_{2}} \cdots \mathbf{e}_{\beta_{m}}
$$

Gelfand et al. showed in [11] that

$$
N S y m \cong \mathbb{K}\left\langle\mathbf{h}_{1}, \mathbf{h}_{2}, \ldots\right\rangle
$$

and that for a composition $\alpha=\left(\alpha_{1}, \ldots, \alpha_{\ell}\right)$ if

$$
\begin{aligned}
\mathbf{e}_{\alpha} & =\mathbf{e}_{\alpha_{1}} \cdots \mathbf{e}_{\alpha_{\ell}} \\
\mathbf{h}_{\alpha} & =\mathbf{h}_{\alpha_{1}} \cdots \mathbf{h}_{\alpha_{\ell}}
\end{aligned}
$$

then $N_{S y m}{ }^{n}$ for $n>0$ has either $\left\{\mathbf{e}_{\alpha}\right\}_{\alpha \models n}$ or $\left\{\mathbf{h}_{\alpha}\right\}_{\alpha \models n}$ as a $\mathbb{K}$-basis. Another basis for $\mathrm{NSym}^{n}$ is the basis of noncommutative ribbon Schur functions $\left\{\mathbf{r}_{\alpha}\right\}_{\alpha \vDash n}$, defined for every composition $\alpha=\left(\alpha_{1}, \ldots, \alpha_{\ell}\right)$ to be

$$
\mathbf{r}_{\alpha}=(-1)^{\ell} \sum_{\alpha \preccurlyeq\left(\beta_{1}, \ldots, \beta_{k}\right)}(-1)^{k} \mathbf{h}_{\beta_{1}} \cdots \mathbf{h}_{\beta_{k}} .
$$

It was shown in [11, Section 6] that QSym is the graded Hopf dual to NSym via the pairing of dual bases

$$
\left\langle M_{\alpha}, \mathbf{h}_{\beta}\right\rangle=\left\langle F_{\alpha}, \mathbf{r}_{\beta}\right\rangle=\delta_{\alpha \beta}
$$

where $\delta_{\alpha \beta}=1$ if $\alpha=\beta$ and 0 otherwise. Meanwhile in [19] it was shown that QSym is the graded Hopf algebra dual to $\mathcal{D}$. Therefore NSym and $\mathcal{D}$ 
are isomorphic. One such isomorphism

$$
\psi: \mathcal{D} \rightarrow \text { NSym }
$$

is given by $\psi\left(B_{\alpha}\right)=\mathbf{h}_{\alpha}$, or equivalently $\psi\left(D_{\alpha}\right)=\mathbf{r}_{\alpha}$. There is also a homomorphism of algebras

$$
\Theta: N S y m \rightarrow \text { Sym }
$$

called the forgetful map, which is given by $\Theta\left(\mathbf{h}_{r}\right)=h_{r}$.

In [3] the authors introduced a new $\mathbb{Z}$-basis for $N_{S y m}^{n}$ for $n>0$, termed the basis of noncommutative Schur functions, which we denote by $\left\{\check{\mathbf{s}}_{\alpha}\right\}_{\alpha \models n}$ and can be defined algebraically as follows.

Definition 5.1. [17, Definition 5.5.1] Let $\alpha, \beta \vDash n$. Then the noncommutative Schur function $\check{\mathbf{s}}_{\beta}$ is defined by

$$
\left\langle\check{\mathcal{S}}_{\alpha}, \check{\mathbf{s}}_{\beta}\right\rangle=\delta_{\alpha \beta} .
$$

There is an involutive anti-automorphism of NSym which maps $\mathbf{r}_{\alpha} \mapsto$ $\mathbf{r}_{\alpha^{*}}$. We define the image of the basis $\left\{\check{\mathbf{s}}_{\alpha}\right\}_{\alpha \models n}$ under this involution by $\check{\mathbf{s}}_{\alpha^{*}} \mapsto \hat{\mathbf{s}}_{\alpha}$ and note by construction that this new basis $\left\{\hat{\mathbf{s}}_{\alpha}\right\}_{\alpha \models n}$, termed the basis of Young noncommutative Schur functions, can be defined algebraically as follows.

Definition 5.2. [17, Definition 5.6.1] Let $\alpha, \beta \vDash n$. Then the Young noncommutative Schur function $\hat{\mathbf{s}}_{\beta}$ is defined by

$$
\left\langle\hat{\mathcal{S}}_{\alpha}, \hat{\mathbf{s}}_{\beta}\right\rangle=\delta_{\alpha \beta} .
$$

We can define both these types of functions combinatorially implicitly as every noncommutative ribbon Schur function is a linear combination of (Young) noncommutative Schur functions with nonnegative integer coefficients. Specifically, if $\alpha \vDash n$, then by Equation (4) and Equation (1), respectively Equation (4) and Equation (2), we have

$$
\mathbf{r}_{\alpha}=\sum_{\beta \models n} \hat{d}_{\beta \alpha} \hat{\mathbf{s}}_{\beta}=\sum_{\beta \models n} \hat{d}_{\beta^{*} \alpha^{*}} \check{\mathbf{s}}_{\beta},
$$

where

$$
\hat{d}_{\beta \alpha}=\#\{\tau \in S Y C T s \mid \operatorname{sh}(\tau)=\beta, \operatorname{comp}(\tau)=\alpha\} .
$$


These functions are pre-images of Schur functions under the forgetful map, that is by [17, Equation (5.8)] and [3, Equation (2.12)],

$$
\Theta\left(\hat{\mathbf{s}}_{\alpha}\right)=\Theta\left(\check{\mathbf{s}}_{\alpha}\right)=s_{\widetilde{\alpha}}
$$

\section{Noncommutative character theory}

In his original paper [25] where descent algebras were defined, Solomon showed that there is a ring homomorphism $\theta_{W}$ from the descent algebra of a finite Coxeter group $W$ into $\mathcal{C} \ell(W)$. In the case of type $\mathrm{A}, \theta_{n}: \mathcal{D}_{n} \rightarrow \mathcal{C} \ell\left(\mathfrak{S}_{n}\right)$ is surjective; specifically, $\theta_{n}\left(B_{\alpha}\right)=\xi^{\alpha}$ for $\alpha \vDash n$.

The maps $\theta_{n}$ collectively extend to a homomorphism of graded algebras $\theta: \mathcal{D} \rightarrow \mathcal{C} \ell[5$, Theorem 1.2, Corollary 7.6]. Now for a composition $\alpha$

$$
\operatorname{ch}\left(\theta\left(B_{\alpha}\right)\right)=\operatorname{ch}\left(\xi^{\alpha}\right)=h_{\widetilde{\alpha}}=\Theta\left(\mathbf{h}_{\alpha}\right)=\Theta\left(\psi\left(B_{\alpha}\right)\right)
$$

that is,

$$
\theta=\operatorname{ch}^{-1} \circ \Theta \circ \psi
$$

This can be described by the following commutative diagram.

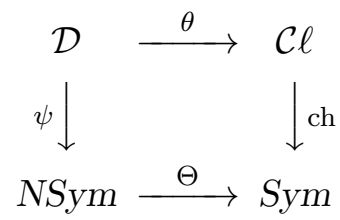

The map $\theta$ extends to a homomorphism of graded algebras $\theta: \mathcal{P} \rightarrow$ $\mathcal{C} \ell$. We refer the reader to [5, Chapter 7] for the details. Recall that the irreducible characters $\left\{\chi^{\lambda}\right\}$ of the symmetric groups form a $\mathbb{Z}$-basis of $\mathcal{C} \ell$, and every character of a symmetric group is a positive $\mathbb{Z}$-linear combination of irreducible characters. We now come to a key definition.

Definition 6.1. [5, p. 13] Any inverse image under $\theta$ in $\mathcal{P}$ of a character $\chi$ afforded by the $\mathfrak{S}_{n}$-module $M$ is called a noncommutative character corresponding to $\chi$, or $M$.

At this point we have collected all our tools and made further useful deductions in the process. As a consequence, our main theorem is now straightforward to prove. 
Theorem 6.2. The bases of $\mathrm{NSym}^{n}$ of Young noncommutative Schur functions $\left\{\hat{\mathbf{s}}_{\alpha}\right\}_{\alpha \models n}$ and of noncommutative Schur functions $\left\{\check{\mathbf{s}}_{\alpha}\right\}_{\alpha \models n}$ are each isomorphic to a set of noncommutative irreducible characters of $\mathfrak{S}_{n}$.

More precisely, every element of the set

$$
\left\{\psi^{-1}\left(\hat{\mathbf{s}}_{\alpha}\right) \mid \alpha \vDash n\right\}
$$

is a noncommutative irreducible character of $\mathfrak{S}_{n}$. Similarly, every element of the set

$$
\left\{\psi^{-1}\left(\check{\mathbf{s}}_{\alpha}\right) \mid \alpha \vDash n\right\}
$$

is a noncommutative irreducible character of $\mathfrak{S}_{n}$.

Proof. Let $\alpha \vDash n, \lambda=\widetilde{\alpha} \vdash n$ and $\chi^{\lambda}$ be the corresponding irreducible character of $\mathfrak{S}_{n}$. By Equation (3)

$$
\operatorname{ch}\left(\chi^{\lambda}\right)=s_{\lambda}
$$

and by Equation (9)

$$
\Theta\left(\hat{\mathbf{s}}_{\alpha}\right)=s_{\lambda} .
$$

Therefore, since by Equation (10) $\theta=\mathrm{ch}^{-1} \circ \Theta \circ \psi$, and so $\theta \circ \psi^{-1}=\mathrm{ch}^{-1} \circ \Theta$ we have that

$$
\theta\left(\psi^{-1}\left(\hat{\mathbf{s}}_{\alpha}\right)\right)=\operatorname{ch}^{-1}\left(\Theta\left(\hat{\mathbf{s}}_{\alpha}\right)\right)=\operatorname{ch}^{-1}\left(s_{\lambda}\right)=\chi^{\lambda} .
$$

Similarly by Equation (3) and Equation (9) we have that

$$
\theta\left(\psi^{-1}\left(\check{\mathbf{s}}_{\alpha}\right)\right)=\chi^{\lambda}
$$

and therefore the theorem follows by Definition 6.1.

Remark 6.3. Both these bases for $\mathrm{NSym}^{n}$ satisfy the strong property that every basis element is isomorphic to a noncommutative irreducible character of $\mathfrak{S}_{n}$. One might ask the question of which bases $\left\{\mathbf{b}_{\alpha}\right\}_{\alpha \models n}$ of NSym ${ }^{n}$ satisfy the weaker condition that some subset of the basis is isomorphic to a set of noncommutative irreducible characters of $\mathfrak{S}_{n}$. As we can see from the above proof a necessary and sufficient condition for this is that there exists for every partition $\lambda \vdash n$ at least one $\alpha \vDash n$ such that

$$
\Theta\left(\mathbf{b}_{\alpha}\right)=s_{\lambda} .
$$

To this end, the "immaculate" basis of $\mathrm{NSym}^{n},\left\{I_{\alpha}\right\}_{\alpha \models n}$, satisfies $\Theta\left(I_{\lambda}\right)=s_{\lambda}$ [2, Corollary 3.30], and so is an example of a basis that satisfies this weaker condition. 


\section{Acknowledgements}

The author would like to thank Kurt Luoto for helpful conversations.

\section{References}

[1] P. Baumann and C. Hohlweg (2008). A Solomon descent theory for the wreath products $G \prec \mathfrak{S}_{n}$. Trans. Amer. Math. Soc. 360 1475-1538. MR2357703

[2] C. Berg, N. Bergeron, F. Saliola, L. Serrano and M. Zabrocki (2012). A lift of the Schur and Hall-Littlewood bases to non-commutative symmetric functions. arXiv:1208.5191.

[3] C. Bessenrodt, K. Luoto and S. van Willigenburg (2011). Skew quasisymmetric Schur functions and noncommutative Schur functions. Adv. Math. 226 4492-4532. MR2770457

[4] D. Blessenohl, C. Hohlweg and M. Schocker (2005). A symmetry of the descent algebra of a finite Coxeter group. Adv. Math. 193 416-437. MR2137290

[5] D. Blessenohl and M. Schocker (2005). Noncommutative character theory of the symmetric group. Imperial College Press. MR2424338

[6] C. Bonnafé and C. Hohlweg (2006). Generalized descent algebra and construction of irreducible characters of hyperoctahedral groups. Ann. Inst. Fourier (Grenoble) 56 131-181. MR2228684

[7] C. Curtis and I. Reiner (2006). Representation theory of finite groups and associative algebras. AMS Chelsea Publishing. MR2215618

[8] G. Duchamp, F. Hivert and J.-Y. Thibon (2002). Noncommutative symmetric functions VI: Free quasi-symmetric functions and related algebras. Internat. J. Algebra Comput. 12 671-717. MR1935570

[9] W. Fulton (1997). Young tableaux. Cambridge University Press. MR1464693

[10] A. Garsia and C. Reutenauer (1989). A decomposition of Solomon's descent algebra. Adv. Math. 77 189-262. MR1020585

[11] I. Gelfand, D. Krob, A. Lascoux, B. Leclerc, V. Retakh and J.-Y. Thibon (1995). Noncommutative symmetric functions. Adv. Math. 112 218-348. MR1327096 
[12] J. Haglund, M. Haiman and N. Loehr (2005). A combinatorial formula for Macdonald polynomials. J. Amer. Math. Soc. 18 735-761. MR2138143

[13] J. Haglund, K. Luoto, S. Mason and S. van Willigenburg (2011). Quasisymmetric Schur functions. J. Combin. Theory Ser. A 118 463-490. MR2739497

[14] J. Haglund, K. Luoto, S. Mason and S. van Willigenburg (2011). Refinements of the Littlewood-Richardson rule. Trans. Amer. Math. Soc. 363 1665-1686. MR2737282

[15] A. Jöllenbeck (1999). Nichtkommutative Charaktertheorie der symmetrischen Gruppen. Bayreuther Math. Schr. 56 1-41.

[16] B. Leclerc, T. Scharf and J.-Y. Thibon (1994). Noncommutative cyclic characters of symmetric groups. J. Combin. Theory Ser. A 75 55-69. MR1395759

[17] K. Luoto, S. Mykytiuk and S. van Willigenburg (2013). An introduction to quasisymmetric Schur functions: Hopf algebras, quasisymmetric functions and Young composition tableaux. Springer. MR3097867

[18] I. Macdonald (1998). Symmetric functions and Hall polynomials. 2nd ed. Oxford University Press. MR1354144

[19] C. Malvenuto and C. Reutenauer (1995). Duality between quasisymmetric functions and the Solomon descent algebra. J. Algebra 177 967-982. MR1358493

[20] R. Mantaci and C. Reutenauer (1995). A generalization of Solomon's algebra for hyperoctahedral groups and other wreath products. Comm. Algebra 23 27-56. MR1311773

[21] S. Mason (2006). A decomposition of Schur functions and an analogue of the Robinson-Schensted-Knuth algorithm. Sém. Lothar. Combin. 57 B57e. MR2461993

[22] S. Poirier and C. Reutenauer (1995). Hopf algebras of tableaux (Algèbres de Hopf de tableaux). Ann. Sci. Math. Québec 19 79-90. MR1334836

[23] B. Sagan (2001). The symmetric group. Representations, combinatorial algorithms, and symmetric functions. 2nd ed. Springer. MR1824028

[24] I. Schur (1901). Über eine Klasse von Matrizen, die sich einer gegebenen Matrix zuordnen lassen. Inaugural-Dissertation Berlin. 
[25] L. Solomon (1976). A Mackey formula in the group ring of a Coxeter group. J. Algebra 41 255-264. MR0444756

[26] R. Stanley (1999). Enumerative combinatorics, volume 2. Cambridge University Press. MR1676282

[27] J.-Y. Thibon (2001). Lectures on noncommutative symmetric functions. In Interaction of combinatorics and representation theory. MSJ Mem. 11 39-94. MR1862149

Stephanie van Willigenburg Department of Mathematics University of British Columbia VANCOUVER, BC V6T 1Z2

CANADA

E-mail address: steph@math.ubc.ca

Received July 6, 2013 